\title{
Ejemplificación del proceso metodológico de la teoría fundamentada
}

\author{
AN EXAMPLE OF METHODOLOGICAL PROCESS OF GROUNDED THEORY
}

Miguel Ángel Bonilla-García (miguel012580@hotmail.com) Instituto de Investigaciones Psicológicas, Universidad Veracruzana (Xalapa, México) ORCID: 0000-0002-1330-5298

Ana Delia López-Suárez (anadelia_lopez@yahoo.com) Instituto de Investigaciones Psicológicas, Universidad Veracruzana (Xalapa, México) ORCID: 0000-0002-1457-7471

\begin{abstract}
Grounded theory, a research method born out of the social sciences field, offers a flexible technique that allows simultaneous data collection and processing. Researchers using this method immerse themselves in an area of study, focusing their observations on the data and taking into consideration not only their own interpretations, but also those of the other subjects involved, in order to strengthen their understanding of the social phenomena under examination. This text briefly describes the concept of grounded theory and uses concrete examples to illustrate how data are analysed from the standpoint of the stereotype as an object of study.
\end{abstract}

Key words: grounded theory, method, technique, coding, analysis.

\section{Resumen}

La teoría fundamentada, nacida al interior de las ciencias sociales, es un método de investigación que posee una técnica flexible y que realiza simultáneamente la recolección y el procesamiento de los datos. En este método, el investigador se encuentra inmerso en el campo de estudio y sus observaciones se abocan al dato, considerando tanto su interpretación como la de los otros sujetos implicados, con la finalidad de fortalecer la comprensión del fenómeno social investigado. En este texto se describe brevemente la teoría fundamentada y cómo se analizan los datos mediante ejemplos concretos, que se presentan a partir del estereotipo como objeto de estudio.

Palabras clave: teoría fundamentada, método, técnica, codificación, análisis.

\section{Introducción}

El principal propósito de la ciencia se ve reflejado en su desarrollo y consiste básicamente en "mejorar la calidad de vida del ser humano" (Delgado 2012:1), mediante de los procesos de investigación que generan conocimiento. El conocimiento es un producto mental nuevo que se genera gracias a "un proceso en el cual se relacionan un sujeto cognoscente (quien conoce) con un objeto de conocimiento (aquello que se conoce)" (Martínez Rodríguez 2011:2). 
El objeto de conocimiento o de estudio se constituye como tal cuando ocurren dos fenómenos: la problematización y la indagación. La primera se refiere a la identificación del objeto de estudio como un problema para un grupo social determinado y la indagación, al problema que merece investigarse. Para abordar al objeto de conocimiento ya problematizado, se requiere de un método de investigación que se sustente en una teoría que suministre la base y el modelo para resolver dicho problema, esto es: el paradigma.

El método de investigación, entonces, se integra "de un tipo particular de técnicas de recolección y análisis de información" (Páramo y Otálvaro 2006:2) y se identifica nominalmente como cualitativo o cuantitativo, aunque también puede haber una mezcla de investigación realizada con un método y con otro (métodos mixtos), los cuales "en combinación proveen de una mejor comprensión de los problemas de investigación" (Creswell y Plano Clark 2007:5), que si se abordaran bajo un método únicamente.

La postura de una investigación no radica en la diferencia de los métodos de investigación, sino en su fundamento ontológico y epistemológico, es decir, en la manera de aproximarse a conocer el objeto de estudio. La ontología, por ejemplo, nos da la visión sobre la naturaleza de la realidad, mientras que la epistemología indica cómo se genera el conocimiento. Por ello: "A todo investigador le debe quedar claro cuáles son los elementos básicos necesarios para realizar la investigación” (Dorantes Rodríguez 2010:13), puesto que éstos tendrán como fin último la creación del conocimiento.

La postura cuantitativa se sustenta en el positivismo, en el cual se busca la objetividad mediante la cuantificación y la medición, con la finalidad de ganar generalización en los hallazgos. Por su parte, la postura cualitativa indaga "en situaciones naturales, intentando dar sentido o interpretar los fenómenos en los términos del significado que las personas les otorgan" (Vasilachis 2006:24). Asimismo, es interpretativa porque otorga valor a las significaciones que tienen los sujetos acerca del objeto de conocimiento, con lo cual se puede llegar al desarrollo de un concepto, un modelo o una teoría.

En este texto se explica la teoría fundamentada (en adelante TF), abarcando los fundamentos de este método de investigación, así como las bases para entender el proceso de la recolección, la codificación y el análisis de los datos. Posteriormente, se ejemplifica su aplicación en una investigación realizada acerca del estereotipo de un candidato idóneo a presidente municipal, para lo cual se explica qué son los estereotipos, las generalidades de la investigación y cómo se llevaron a cabo la codificación abierta, la generación de familias, la codificación axial y la codificación selectiva.

\section{La teoría fundamentada (TF)}

La TF es un método de investigación cuyo soporte epistemológico radica en la vinculación entre un sujeto que busca la comprensión de un objeto a investigar mediante "las acciones y significaciones de los participantes de la investigación" (Charmaz 2013:272). Lo anterior implica que el investigador "recoge, codifica y analiza datos en forma simultánea" (Soneira 2006:155) mas no sucesiva. En ello radica la singularidad de la TF como un proceso metódico, sistemático e interpretativo, propio del paradigma cualitativo.

El inicio de la TF se remonta al año de 1967. Sus creadores fueron B. Glaser y A. Strauss. En esta primera época de la TF, conocida como escuela clásica u ortodoxa, se combinan elementos cuantitativos como el empirismo cuantitativo y cualitativos como el interaccionismo simbólico (reinterpretación y redefinición de significados por parte de los sujetos), con la finalidad de construir teoría. La segunda época de la TF es 
propuesta por el mismo A. Strauss y por J. Corbin. Su perspectiva se conoce como la escuela reformulada, la cual rompió con la clásica, al impulsar de manera significativa al interaccionismo simbólico, lo que permitió la introducción de la TF a diversas disciplinas del saber, como fue el caso de la psicología.

En el actual siglo XXI, una tercera escuela tiene un fuerte auge. Ésta es la constructivista que planteó K. Charmaz. Su propuesta es un rediseño al modelo positivista, cuestionando sus bases objetivistas, mediante un enfoque sistemático que fomenta la integración de (1) la experiencia subjetiva del investigador, como prioridad y (2) las condiciones sociales propias del objeto de estudio (Charmaz 2013).

La escuela constructivista hace énfasis en el objeto de estudio, al cual también llama fenómeno, pero deja en un segundo plano a los métodos para estudiarlo. Esta postura se sustenta en que el carácter cualitativo de la TF debe evitar las prácticas positivistas, teniendo claro que se buscan las representaciones interpretativas de la realidad social (la cual es diferente según el ambiente o contexto), en vez de informes que tengan un sello de una pretensión puramente objetivista.

La TF es un método que implica la recolección y el análisis simultáneo de datos; se caracteriza por ser flexible, ya que se pueden combinar métodos cuantitativos y cualitativos, además de diferentes técnicas de investigación, cuyo análisis y contraste permiten la triangulación metódica, o bien, pueden realizarse composiciones metodológicas apropiadas para abordar al objeto de estudio y generar teoría acerca del mismo. La finalidad principal de la TF es la generación de teoría, por lo que "los métodos son un medio para conseguir un fin, no el fin en sí mismo" (Andréu Abela et. al. 2007:54).

En el proceso metodológico de la TF intervienen dos grandes estrategias: el método de comparación constante y el muestreo teórico. Según Soneira (2006), el método de comparación constante expresa por sí mismo la flexibilidad de la TF e implica por parte del investigador (1) la recolección, (2) la codificación y (3) el análisis de los datos, en forma simultánea. Lo anterior se explicará en el siguiente apartado.

Con el muestreo teórico se descubren las características o atributos de la categoría; también se verifican las semejanzas y las diferencias de dichas propiedades y se sugieren interrelaciones entre ellas para la generación de la teoría. Asimismo, el muestreo teórico representa a la muestra de la investigación ya definida, es decir, al total de participantes de la investigación, determinado por la saturación teórica. "En el instante en que las comparaciones constantes entre los datos no evidencian nuevas relaciones o propiedades de los datos; la riqueza que representan los datos se comienza a agotar. En ese momento se evidencia la saturación teórica, donde el muestreo teórico comienza a definir su término, por lo tanto, no es conveniente realizar más entrevistas y observaciones. Los datos comienzan a tornarse repetitivos y no se obtiene nada nuevo" (San Martín Cantero 2014:113).

\section{La recolección, la codificación y el análisis de los datos}

El proceso metodológico de la TF inicia con la elección de la técnica y del instrumento que el investigador considere adecuados; se ingresa a campo y se recaban los datos. La recolección se realiza a través de dos procedimientos: el ajuste y el funcionamiento. El ajuste es la generación de categorías conceptuales a partir de los datos y el funcionamiento es la capacidad de las categorías conceptuales para explicar lo investigado.

En los datos se identifican sus atributos, es decir, las cualidades o las características del dato, que se comparan entre sí para encontrar coincidencias y distinguir diferencias. Los datos que comparten las 
mismas características se agrupan; se les asigna un rótulo o un nombre determinado que indica el concepto al que pertenecen, es decir, se congregan en un mismo código (según el nombre que reciben en el software de análisis cualitativo) o subcategoría. El proceso de asignar códigos se llama codificación.

La codificación es un proceso sistemático que contiene tres tipos de codificación: (1) abierta, (2) axial y (3) selectiva. En la codificación abierta se generan códigos a partir de dos fuentes: la pre-codificación y los códigos in vivo. La pre-codificación son los códigos o subcategorías que se generan gracias a la subjetividad inductiva del investigador, mientras que los códigos in vivo son las expresiones y el lenguaje de los participantes, encontradas en las frases literales que emplearon y cuya riqueza se perdería al ubicarlas dentro de un código o porque simplemente no existe un rótulo que la abrevie.

El ATLAS.ti, un software especializado para la elaboración de TF, también hace la diferencia de los códigos antes explicados y los códigos libres, los cuales están previamente identificados dada la teoría existente (deducción). La aportación del paradigma cuantitativo radica en que los códigos pueden contabilizarse sacando la frecuencia de aparición de los códigos.

Al igual que se hace la agrupación de los datos, los códigos o subcategorías se organizan para conformar una organización más grande llamada familia (según el software) o categoría. Se analiza el contenido de cada código, comparándolo con los demás e identificando qué tienen en común y se decide si pertenecen a la misma clase de respuestas, nombrando al grupo al que pertenecen. Esto es semejante a la comparación de datos para ubicarlos en un código, pero en un nivel superior, como si fuera una agrupación de personas que conforman una ciudad, ciudades que conforman estados, estados que conforman países.

A partir de la codificación abierta se realiza la codificación axial. Ésta consiste en la búsqueda activa y sistemática de la relación que guardan los códigos y las familias (o subcategorías y categorías, respectivamente) entre sí. Las relaciones posibles que se pueden establecer se encuentran en el Cuadro 1. El tipo de relación que guardan unos con otros resulta de la comparación constante entre códigos y familias, es decir, por el contraste de las semejanzas y de las diferencias existentes. La categoría que tiene mayor número de relaciones con las demás se llama categoría central.

\section{Cuadro 1. Nomenclatura de relaciones en ATLAS.ti}

\begin{tabular}{|c|c|}
\hline Nomenclatura & Función de la relación \\
\hline$==$ & Se asocia con \\
\hline[] & Es parte de \\
\hline$=>$ & Es causa de \\
\hline$<>$ & Contradice a \\
\hline isa & Es una \\
\hline$:$ & Sin nombre \\
\hline$*\}$ & Es propiedad de \\
\hline
\end{tabular}

Fuente: Elaboración propia (Software ATLAS.ti Versión 7)

Finalmente, la codificación selectiva es la relación conceptual y teórica que guardan entre sí los códigos o familias y que se concreta en la teorización. Ésta última ocurre cuando el investigador integra dichas relaciones dentro de un relato que contiene un conjunto de proposiciones. Una proposición es un enunciado que expresa la relación entre dos o más categorías y sus propiedades. Aquí el investigador emplea su habilidad para discernir, comprender y dar sentido a los datos, lo cual es conocido como sensibilidad teórica. 
La teoría que resulta de los datos se clasifica en sustantiva y en emergente. La sustantiva se genera durante el proceso de interacción del sujeto cognoscente en el campo donde se encuentra el fenómeno de estudio, con su acercamiento a los datos a través de los participantes. La emergente tiene que ver con la recolección de los datos y con su comparación cuando éstos van surgiendo. Esa comparación de datos (análisis teórico) permite construir relaciones entre categorías para explicarlas, lo cual es en sí mismo la generación de una teoría.

La recomendación de los expertos, para la aplicación de la TF, es apegarse a alguna de las tres escuelas existentes, antes descritas. Debe unirse a aquélla que el investigador considere adecuada para la comprensión del objeto de estudio. Para esto es indispensable tener claridad en las características de la población y en las del ambiente de estudio.

El proceso metodológico aquí descrito se aproxima a la escuela reformulada y a la constructivista. El ejemplo, que más adelante se presenta, partió de la conceptualización previa del constructo, lo que permitió ser preciso en el análisis de los datos. Es importante recordar que, en la investigación cualitativa, específicamente en la TF, no siempre es posible partir de cero en el conocimiento del fenómeno, ni puede basarse únicamente en la sensibilidad teórica del investigador la cual "no se refiere solamente a la capacidad para conceptualizar y formular teoría apenas emerja de los datos... sino también a la capacidad de discernir teóricamente dentro de un área específica de investigación" (Jones et. al. 2004:51). En razón de lo anterior, se considera que la investigación regularmente requiere una mínima revisión de la teoría.

\section{Los estereotipos}

Previo a definir al estereotipo, es importante anotar que forma parte de "los procesos mentales mediante los cuales la gente conoce al mundo social" (Páez et. al. 1994:128) y que la psicología llama cognición social. También se define como "las maneras en que interpretamos, analizamos, recordamos y empleamos la información sobre el mundo social -en otras palabras, cómo pensamos acerca de los demás" (Baron y Byrne 2005:82).

Los estereotipos son "creencias consensuales sobre los atributos de un grupo social y sus miembros... Se trata de atribuciones socialmente compartidas sobre las características de personalidad, las conductas y/o los valores de un colectivo y de sus miembros sin atención a las diferencias intragrupales" (Smith y Pérez 2007:130). También pueden definirse como un conjunto de creencias positivas o negativas que ideamos acerca de grupos sociales. Son creencias sobre características particulares "que nos forjamos respecto de ciertos grupos y que influyen sobre los juicios que hacemos acerca de los individuos que los conforman" (Kassin et. al. 2013:130), es decir, generalizamos a todo el grupo las características que atribuimos a ciertos integrantes.

Aunque existen diferentes definiciones, "parece existir consenso en que son esquemas cognitivos" (Gómez Jiménez 2007:14). Los estereotipos se forman como un esquema mental, pero también son "sociales no solo porque son el resultado de influencias sociales sobre el individuo, sino porque pueden convertirse a su vez en especie de norma social" (Rodríguez y Moya 1998:28). En este sentido Gómez Jiménez y Rodríguez y Moya, parafraseando los hallazgos de Henri Tajfel, reiteran que los estereotipos además de fungir como norma social, también ayudan a mantener funciones sociales o grupales, tales como (1) tener una identidad positiva, (2) justificar las acciones del grupo con respecto de otros grupos y (3) explicar la realidad social. 
Explicado coloquialmente, en la vida diaria convivimos con una variedad de personas, que pueden pertenecer a nuestro grupo social o a otros. Para emitir juicios o una conducta determinada ante lo que vivimos, requerimos comprender el mundo y adaptarnos a él. Las experiencias sociales que hemos tenido aportan información compleja que sería muy difícil de manejar si no la redujéramos. Precisamente, estereotipar es uno de los procesos mentales que nos permite reducir la cantidad de información acerca de los grupos sociales, para tener una postura frente a alguien y su función es ahorrar energía cognoscitiva. Ese ahorro se hace evidente cuando observamos unas cuantas características de alguien y determinamos a qué grupo pertenece, ubicándolo entonces dentro de cierta categoría o del grupo social que presenta la característica observada. Si un niño lleva al hombro una mochila suponemos que es un estudiante y no un traficante de drogas, por ejemplo. Eso nos lleva a no considerarlo como una amenaza y a no tener miedo cuando pasamos frente a él.

Kassin et. al. (2013) presentan el caso de los bomberos de raza de color en la sociedad estadounidense. Si la casa de una persona se incendiara y llegara un bombero de raza negra, la persona afectada lo categorizaría como bombero y no por su raza, en cambio, si estuvieran en una fiesta, se le categorizaría más por su raza que por ser bombero. En este ejemplo se identifican dos grupos de referencia: el grupo de trabajo y el grupo étnico. A partir de un aspecto de la persona que percibimos en determinado contexto, la ubicamos dentro de una categoría (estereotipamos) y emitimos juicios que determinan nuestro comportamiento. De esa manera, nuestro cerebro toma el camino corto para ahorrar energía cognitiva.

\section{Aplicación de la TF al estudio del estereotipo}

En este apartado se presenta la aplicación metodológica de la TF al estudio del estereotipo de un candidato idóneo a presidente municipal, el cual se definió como el conjunto de atributos que debería poseer un candidato, según la percepción social y las expectativas comunitarias. Se tuvo entonces como propósito indagar los atributos deseables y los no deseables de un candidato idóneo a presidente municipal. Los atributos no deseables se consideraron únicamente para incrementar las respuestas de las personas, ya que muchas veces es difícil evocar el rasgo positivo o la presencia de atributos; para algunas personas quizá es más sencillo expresar lo que no debería ser o lo que no se quiere en un candidato.

Los participantes fueron ciudadanos de uno de los 212 municipios del Estado de Veracruz, México. El estudio se realizó en un "ámbito público político" (Fraser 1993:49). Recibe esos calificativos porque el ambiente político está inmerso en un gran ambiente que es el público.

Los datos se recolectaron mediante las técnicas de cuestionario y de entrevista. Se preguntó: ¿cuáles son las características que debe tener un candidato a presidente municipal por este municipio? y ¿qué características tendría un candidato a la presidencia municipal por el cual no votarías? Los datos se transcribieron e incorporaron al software ATLAS.ti (versión 7), conforme se iban recolectando.

\section{La codificación abierta}

El análisis inició con la comparación de los datos, a partir de la interpretación del investigador acerca del mundo de los participantes. En las respuestas se identificó la idea principal y se generó un código. Además, se realizó un análisis de afinidad semántica, que consistió en verificar en un diccionario los significados usuales de las palabras que aparecían en esas ideas principales, se contrastaron sus significados y se generaron los códigos. Por ejemplo, se determinó que honesto y honrado eran sinónimos y se agruparon en el código honesto. 
En el caso de los atributos no deseables, se ubicó el código en un primer momento, para después encontrar el antónimo con la ayuda de un diccionario, de tal manera que pudiera hablarse de presencia de atributos y no de su ausencia. Por ejemplo, para el código deshonesto se empleó el antónimo honesto.

A continuación, se presentan algunas respuestas de los participantes para ejemplificar la generación de códigos. Ante la pregunta de los atributos deseables, las personas respondieron lo siguiente acerca de cómo debería ser el candidato: "honesto, sencillo, con aspiraciones a mejorar la comunidad", "que sea social con el pueblo", "que sea amable con todo el pueblo y no cambie cuando llegue al puesto". El dato honesto se clasificó en el código honesto. Las respuestas: sencillo, que sea amable con todo el pueblo y no cambie cuando llegue al puesto se clasificaron en el código sencillo. La respuesta con aspiraciones a mejorar la comunidad se identificó con el código progresista y la afirmación que sea social con el pueblo se ubicó con el código sociable.

Algunas respuestas para la pregunta de los atributos indeseables fueron las siguientes: “De los que siempre mienten y dicen servir al pueblo y se sirven del pueblo". Esta respuesta caracteriza a una persona deshonesta, al utilizar el antónimo, se clasificó en el código honesto. "Que no caiga (bien), que no invite, que no salude" se clasificaron por su antónimo en el código sociable. "Que fuera muy engrandecido y al llegar al puesto alzarse más” se clasificó por su antónimo en el código sencillo.

Las definiciones de los códigos antes mencionados fueron las siguientes:

Honesto: Persona que actúa con integridad, que tiene una administración correcta en lo económico y en los bienes, y que cumple su palabra o sus promesas.

Sencillo: Persona que se comporta de manera amable y educada, mostrando un trato igualitario y recíproco con la gente.

Progresista: Persona con visión e inclinación hacia la mejoría o el desarrollo comunitario.

Sociable: Persona que tiene la facilidad de entablar buenas relaciones humanas en su comunidad.

Algunas expresiones de los participantes contenían tal riqueza que complicaba su incorporación a alguno de los códigos identificados previamente, por lo que se consideraron códigos in vivo. Por ejemplo: "que éste (el candidato) fuera manejado por terceros" y "que no sea descendiente (el candidato) de personas de poder".

En ocasiones se genera un código a partir de algunas respuestas que son muy parecidas, pero quizá ocurra que al leer una respuesta, el investigador se cuestione qué tan semejante es a las ya clasificadas bajo determinado código. Entonces, éste debe determinar si vale la pena conservar diferencias sutiles 0 desvanecerlas, según el propósito de la investigación. A esto nos referíamos cuando anteriormente hablamos del proceso de comparación constante entre datos, códigos y familias. Es importante detallar las diferencias, porque los códigos y las categorías deben ser mutuamente excluyentes para un mismo nivel de análisis, condición propia de todo proceso de codificación o categorización.

Los códigos y las familias deben definirse claramente por dos razones: para incrementar la precisión en la codificación de manera personal y para que cualquiera empleé los mismos criterios al llevar a cabo este proceso. En algunas investigaciones se tiene a varios codificadores que analizan diferentes segmentos de 
información o bien, hay varias personas que codifican la misma información y posteriormente se verifica el acuerdo entre éstas, para tener mayor confiabilidad en el análisis.

El software de análisis cualitativo también se auxilia de notas u observaciones de quienes analizan los datos en el proceso de investigación, que reciben el nombre de memo. El memo se emplea para precisar lo que se requiera o para conservar las ideas o reflexiones que surgen en la cabeza del investigador.

\section{La generación de familia o categoría}

Los códigos honesto, humilde, sencillo, con sentido de bien común, responsable, diligente, solidario, respetuoso, sociable y trabajador conformaron la familia filosofía moral (ética). Aquí se mencionan únicamente los que aparecieron con mayor frecuencia, pero hubo más códigos dentro de esa familia.

Filosofía moral (ética) se definió como el conjunto de valores morales (virtudes) que un candidato debe poseer para desempeñar su trabajo con calidad humana, por el bien de la comunidad. Para definir esta familia se empleó lo teorizado por Cortina (2013). A continuación, se indica cómo se vinculan los hallazgos de la investigación con el desarrollo teórico preexistente.

La filosofía moral (ética) se refiere a ser moral, es decir, a la manera como actuamos cotidianamente según normas y principios morales dentro del grupo social en el que estamos inmersos. La moral se determina según los códigos de conducta que la legitiman. Un código moral contiene normas sociales aceptables para un grupo social y que permite a un individuo ser aceptado dentro de dicho grupo y, por lo tanto, formar parte de él.

El nombre hace referencia a dos aspectos filosóficos: la ética y los valores. Los valores son indisociables de la ética. La ética está cimentada en la confianza porque "la confianza es el principal «recurso moral» de una sociedad" (Cortina 2013:15). Si bien la ética y los valores son de aplicación universal, en cada grupo social se tienen cánones propios de aplicación.

Los valores son la parte práctica de la ética; concretamente, los valores denominados morales, según Cortina (2013), son los aplicables al ámbito público. Estos valores -por ser morales- ayudan a crear en las personas "un determinado modelo ideal de buena conducta socialmente establecido" (Cortina y Martínez 2008:14), es decir, permiten tener un comportamiento adecuado y socialmente aceptable.

Los valores se legitiman y por ende garantizan confianza, asimismo, se ven como cualidades en determinadas personas $y$, por lo tanto, también se refieren a la contraparte o extremo negativo, que serían los atributos indeseables o defectos. En este sentido podemos decir que "los valores son las interpretaciones más básicas sobre las que se fundamenta una cultura, y que expresan las necesidades idiosincrásicas del tipo de hombre que evalúa ligadas a su conservación y desarrollo" (Sánchez Meca 2013:3).

Los valores son también vistos como virtudes que ayudan a obrar bien, como por ejemplo la justicia, la prudencia, la fortaleza y la honestidad entre muchas otras. Al hablar de obrar bien se hace referencia al carácter de la persona y de la sociedad, vistos como sistemas interdependientes. Esto tiene que ver con el origen etimológico de las palabras moral y ética (mosmoris y ethos) que significan formación del carácter y hábitos que influyen en el obrar con rectitud. Al hablar de carácter, se hace alusión a la manera o forma de ser de las personas inmersas en una sociedad, que legitima valores -elementos inherentes a la moral-, para que ésta funcione adecuadamente. 


\section{La codificación axial}

Durante el proceso de comparación constante se identificaron relaciones entre códigos (subcategorías) y familias (categorías). Por ejemplo, el ser sencillo antecede a ser sociable, por lo que el código sencillo es causa del código sociable. En el caso de la relación entre los códigos honesto y progresista, se tuvo que honesto se asocia con progresista, ya que una persona que cumple sus promesas y que tiene un adecuado manejo en las finanzas, buscará la mejora y el progreso de su comunidad y empleará los recursos necesarios para conseguirlo. Las relaciones también pueden establecerse entre familias.

Los resultados de este análisis son dos: un esquema de relaciones que condensa la teoría e identificar la densidad de un código o de una familia. La densidad es el número de relaciones que tiene con los demás. La familia central en este caso fue la de filosofía moral (ética).

\section{La codificación selectiva}

Por último, se llegó a la codificación selectiva que es en sí misma la teorización. Ésta se presenta a continuación. Cualquier aspirante a presidente municipal debe poseer valores universales y ética en su actuar personal y en su actuar como servidor público. Debe tener valores como honestidad, humildad, amabilidad, sentido de bien común, responsabilidad, diligencia, solidaridad, respeto, sociabilidad y trabajo. La comparación entre datos, en un ambiente político, pone al descubierto necesidades y deberes evidentes. En este caso, el candidato idóneo debe tener ciertos atributos para ser presidente municipal, destacando su obligación de servir con sentido de responsabilidad, ética y diligencia.

\section{Conclusiones}

Las experiencias sociales de cada población y por lo tanto de cada sujeto, permiten que las personas den significados diferentes a los acontecimientos y, por ende, generan mundos muy singulares. Las personas significan sus motivaciones en un vocabulario particular que corresponde a su mundo y a su vez, refiere a la singularidad de contextos sociales, culturales, históricos y económicos. Merleau-Ponty indica que todos "somos amalgamas resultado de nuestras relaciones en y con el mundo; el mundo siempre está con nosotros" (en San Martin Cantero 2014:107). La TF es una de las metodologías que permite estudiar con profundidad estos mundos, ya que considera la interpretación que tienen los sujetos acerca de su entorno.

La comprensión de los fenómenos con suficiente profundidad requiere también de flexibilidad en el método, por lo que se recurre a diferentes técnicas de recolección de información. La flexibilidad también abarca al planteamiento de la investigación. En la TF se puede partir de una base cero en lo teórico o bien, desde los elementos teóricos existentes sobre la temática a investigar. Lo anterior favorece la solidez en la teoría generada, ya que se parte fundamentalmente de la contrastación de los hallazgos y de éstos con la teoría. Como pudo apreciarse en la descripción del ejemplo, la comparación entre datos, entre códigos, entre familias y a su vez con la teoría es un proceso permanente en los métodos de la TF.

Aunque el fundamento epistemológico de la TF le confiere una postura eminentemente cualitativa, se complementa con análisis de frecuencia en torno a la aparición de cada código. Charmaz dice que "los métodos de la teoría fundamentada proporcionaron un modelo para llevar a cabo la investigación cualitativa, sellada con la aprobación positivista" (2013:274), ya que la sistematización con la que se trabajan los datos fue dada por el positivismo. Debe tenerse siempre presente que el fin último de la TF es la generación de teoría: a veces se crea una nueva o se perfecciona alguna ya existente, lo cual es una 
de sus principales aportaciones a la ciencia.

\section{Agradecimientos}

Miguel Bonilla-García es becario para estudios de maestría de la Secretaría de Educación de Veracruz.

\section{Bibliografía}

Andréu Abela, J; García-Nieto, A; Pérez Corbacho, A. M. 2007. Evolución de la teoría fundamentada como técnica de análisis cualitativo. Santiago de Compostela: Centro de Investigaciones Sociológicas (CIS).

Baron, R. A; Byrne, D. 2005. Psicología social. México: Pearson Educación.

Cortina, A. 2013. ¿'Para qué sirve realmente la ética? Madrid: Paidós.

Cortina, A; Martínez, E. 2008. Ética. Madrid: Akai.

Charmaz, K. 2013. La teoría fundamentada en el siglo XXI: Aplicaciones para promover estudios sobre la justicia social, pp. 270-325. En: N. K. Denzin; Y. S. Lincoln (comps.) Estrategias de investigación cualitativa: Vol. III. Buenos Aires: Gedisa.

Creswell, J. W; Plano Clark, V. L. 2007. Designing and conducting mixed methods research. New York: Sage Publications.

Delgado, C. 2012. La teoría fundamentada: decisión entre perspectivas. Bloomington: Author House.

Dorantes Rodríguez, C. H. 2010. El proyecto de investigación en psicología, de su génesis a la publicación. México: Universidad Iberoamericana.

Fraser, N. 1993. Repensar el ámbito público: Una contribución a la crítica de la democracia realmente existente. Debate feminista 7: 23-58. http://www.debatefeminista.pueg.unam.mx/wpcontent/uploads/2016/03/articulos/007_02.pdf

Gómez Jiménez, A. 2007. Estereotipos, pp. 213-241. En: J.F. Morales; M.C. Moya; E. Gaviria; I. Cuadrado. Psicología social. Madrid: McGraw-Hill.

Jones, D; Manzelli, H; Pecheny, M. 2004. Grounded theory: una aplicación de la teoría fundamentada a la salud. Cinta moebio 19: 1-19. http://www.moebio.uchile.cl/19/manzelli.html

Kassin, S; Fein, S; Markus, H.R. 2013. Psicología social. México: Cengage Learning.

Martínez Rodríguez, J. 2011. Métodos de investigación cualitativa. Silogismos de investigación 8(1): 1-43. http://www.cide.edu.co/ojs/index.php/silogismo/article/view/64/53

Páez, D; Marques, J; Insúa, P. 1994. Estructuras y procesos de la cognición social, pp. 123-170. En: J.F. Morales. Psicología social. Madrid: McGraw-Hill. 
Páramo, P. y Otálvaro, G. 2006. Investigación alternativa: por una distinción entre posturas epistemológicas y no entre métodos. Cinta moebio 25:1-7. www.moebio.uchile.cl/25/paramo.html Rodríguez, R; Moya, M.C. 1998. España vista desde Andalucía. Estereotipos e identidad. Psicología política 16: 27-48. http://www.uv.es/garzon/psicologia\%20politica/N16-2.pdf

San Martín Cantero, D. 2014. Teoría fundamentada y ATLAS.ti: recursos metodológicos para la investigación educativa. Revista electrónica de investigación educativa, 16(1): 104-122.

http://redie.uabc.mx/vol16no1/contenido-sanmartin.html

Sánchez Meca, D. 2013. ¿Qué son y cómo se forman los valores? Estudios Nietzsche: Revista de la Sociedad Española de estudios sobre Friedrich Nietzsche 2(13): 1-6.

https://dialnet.unirioja.es/servlet/articulo?codigo=5097671

Smith, V; Pérez, R. 2007. Atribuciones estereotípicas de calidez y habilidad en jóvenes universitarios. Revista de ciencias sociales 117-118: 129-147.

http://revistas.ucr.ac.cr/index.php/sociales/article/view/11019/10399

Soneira, A. J. 2006. La teoría fundamentada en los datos (grounded theory) de Glaser y Strauss, pp. 153173. En: I. Vasilachis. Estrategias de investigación cualitativa. Barcelona: Gedisa.

Vasilachis, I. 2006. Estrategias de investigación cualitativa. Barcelona: Gedisa.

Recibido el 3 Oct 2016

Aceptado el 27 Nov 2016 